\title{
Hepatic accumulation on FDG-PET/CT due to follicular lymphoma
}

\author{
Kosuke Ishizuka $^{1}$, Kiyoshi Shikino ${ }^{1}$, Daiki Yokokawa $^{1}$, and Masatomi Ikusaka ${ }^{1}$ \\ ${ }^{1}$ Chiba University Hospital Department of General Medicine
}

February 10, 2021

\begin{abstract}
Follicular lymphoma is the second most common type of non-Hodgkin's lymphoma. Hepatic accumulation observed on FDG$\mathrm{PET} / \mathrm{CT}$, which is the most useful imaging finding, is observed in $15 \%$ of cases. Secondary hepatic lymphoma is defined based on distant involvement, and it typically presents as diffuse infiltration, as in this case.
\end{abstract}

\section{Hepatic accumulation on FDG-PET/CT due to follicular lymphoma}

Kosuke Ishizuka, $\mathrm{MD}^{1}$, Kiyoshi Shikino, $\mathrm{MD}, \mathrm{PhD}^{1}$, Daiki Yokokawa, $\mathrm{MD}^{1}$, Masatomi Ikusaka, MD, $\mathrm{PhD}^{1}$

${ }^{1}$ Department of General Medicine, Chiba University Hospital, Japan

Article type: Clinical Images

Key words: follicular lymphoma; hepatic accumulation

Word Count: 199 words

Disclosure: The authors declare no conflicts of interest in association with this study.

\section{Corresponding author}

Kosuke Ishizuka, MD

Department of General Medicine, Chiba University Hospital

1-8-1, Inohana, Chuo-ku, Chiba-city, Chiba pref. Japan

Tel. +81-43-222-7171 (Ext. 6438); +81-43-224-4758 (Direct line)

Fax. $+81-43-224-4758$

E-Mail: e103007c@yokohama-cu.ac.jp

Number of figures: 2

Number of references: 2

\section{Key Clinical Message}

Follicular lymphoma is the second most common type of non-Hodgkin's lymphoma. Hepatic accumulation observed on FDG-PET/CT, which is the most useful imaging finding, is observed in $15 \%$ of cases. Secondary hepatic lymphoma is defined based on distant involvement, and it typically presents as diffuse infiltration, as in this case.

\section{Case presentation}


A 67-year-old man presented with a 1-year history of cervical and para-aortic lymph node lymphadenopathy on plain computed tomography (CT) scan and was referred to our hospital. Physical examination revealed no superficial lymphadenopathy and hepatomegaly. Laboratory tests showed elevated C-reactive protein $(\mathrm{CRP}, 11.33 \mathrm{mg} / \mathrm{dL})$ and soluble interleukin-2 receptor (sIL-2R, $1010 \mathrm{U} / \mathrm{mL}$ ) but no other findings including hepatobiliary enzymes. Abdominal ultrasonography showed no abnormalities. The ${ }^{18} \mathrm{~F}$-fluorodeoxyglucose (FDG) positron emission tomography/computed tomography (PET/CT) scan showed multiple sites of FDG uptake in the enlarged cervical (SUVmax, 3.5) and para-aortic lymph nodes (SUVmax, 4.9) (Figure 1). Additionally, diffuse FDG uptake was observed in the liver (SUVmax, 5.7) (Figure 2). Right supraclavicular lymph node biopsy detected follicular lymphoma. Secondary hepatic follicular lymphoma was diagnosed and classified as Lugano system stage IV. Rituximab monotherapy was started. CRP and sIL-2R levels improved. FDG uptake in the enlarged lymph nodes and diffuse FDG uptake in the liver disappeared.

Follicular lymphoma is the second most common type of non-Hodgkin's lymphoma. Hepatic accumulation observed on FDG-PET/CT, which is the most useful imaging finding, is observed in $15 \%$ of cases. ${ }^{1}$ Secondary hepatic lymphoma is defined based on distant involvement ${ }^{2}$, and it typically presents as diffuse infiltration, as in this case. ${ }^{2}$

Funding: None.

Acknowledgements: None.

Author's Contribution: KI, KS, DY, and MI managed the patient. KI wrote the draft. KS, DY, and MI revised this article. The patient provided consent to report the case.

\section{References}

Lee WK, Lau EW, Duddalwar VA, Stanley AJ, Ho YY. Abdominal manifestations of extranodal lymphoma: spectrum of imaging findings. AJR Am J Roentgenol. 2008; 191: 198-206.

Alves AMA, Torres US, Velloni FG, Ribeiro BJ, Tiferes DA, D'Ippolito G. The many faces of primary and secondary hepatic lymphoma: imaging manifestations and diagnostic approach. Radiol Bras. 2019; 52: $325-30$.

\section{Figure Legends}

Figure 1. FDG-PET/CT scan showed FDG uptake in the enlarged cervical lymph nodes (SUVmax, 3.5) (arrow).

Figure 2. FDG-PET/CT scan showed diffuse FDG uptake in the liver (SUVmax, 5.7) (arrow heads).

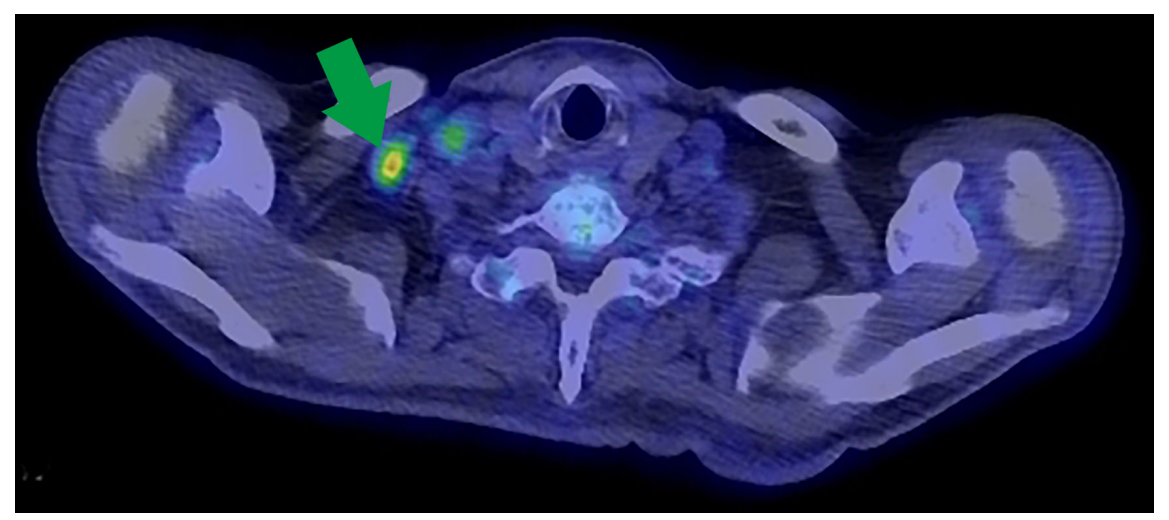




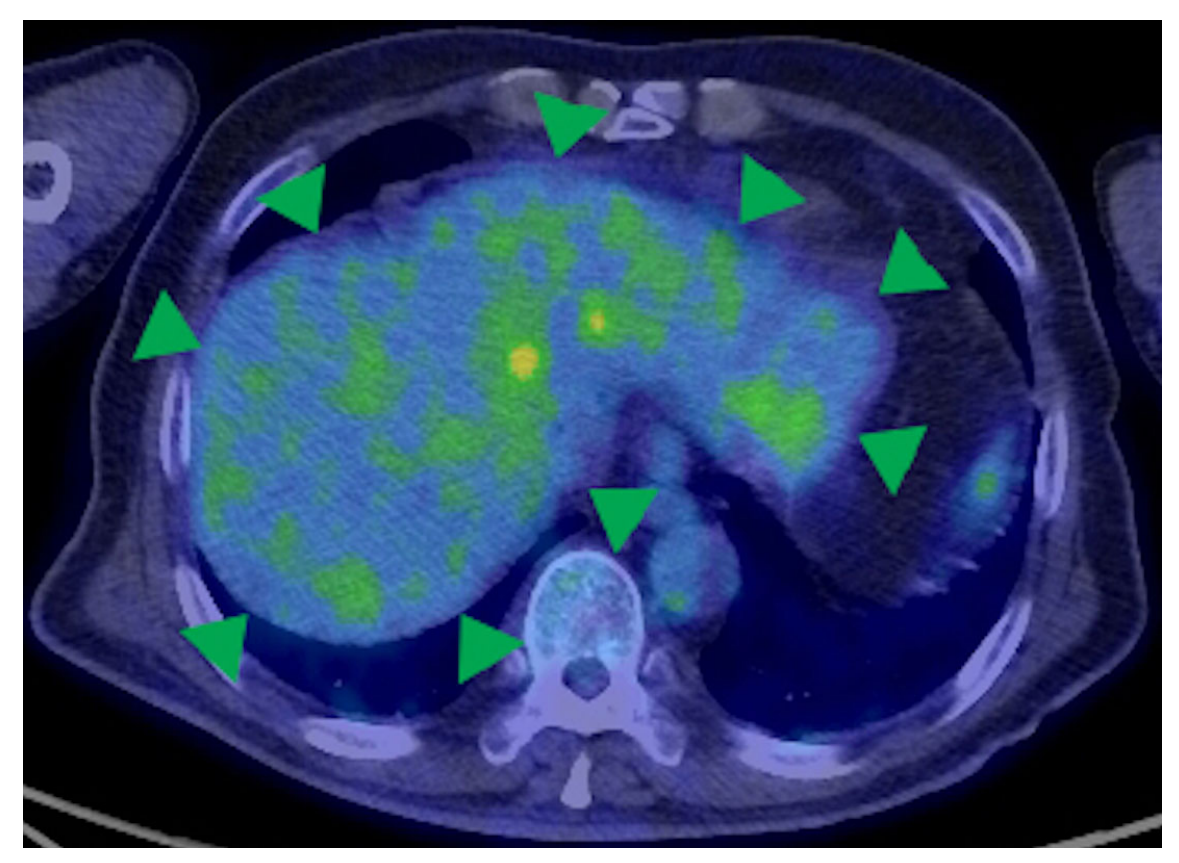

